\title{
Federico Canaccini
}

\section{Essere (filoimperiali) o non essere? Questo è il dilemma}

\author{
Relazioni politiche tra i conti Guidi, Dante Alighieri \\ e l'imperatore Enrico VII a partire dal cosiddetto \\ "trittico Battifolle" (epistole VIII-X)
}

\begin{abstract}
Le tre brevi lettere indirizzate dalla contessa Gherardesca all'imperatrice Margherita di Brabante, attribuite a Dante (epp. VIII-X), pur nella loro esiguità e povertà di contenuto, se analizzate sotto la chiave di lettura politica, mostrano un complicato gioco di poteri in seno alla potente famiglia Guidi, divisa su posizioni diverse in merito alla discesa di Enrico VII. Dalle brevi lettere, infine, emerge l'idea di Dante e la sua speranza nell'avvento di un nuovo corso che però non si avvererà mai.
\end{abstract}

The three short epistles addressed by countess Gherardesca to the Empress Margherita of Brabante, attributed to Dante (epp. VIII-X), despite their size and their insubstantial content, can show, if analyzed from a political point of view, a complex dynamic of power within the powerful family of the counts Guidi, who was divided between different opinion regarding Henry VII's mission in Italy. From the epistles, we can grasp Dante's ideas and his hope of the advent of a new course that will, nonetheless, never come true.

Parole chiave: Dante, Guidi, Epistole, Ghibellini, Enrico VII.

All'interno della produzione dantesca, il cosiddetto "trittico Battifolle" non brilla certamente per originalità, spessore o fama. Eppure, nonostante la povertà di contenuto e l'esiguità testuale, pare che questi «biglietti di poco o punto valore» ${ }^{1}$ possano ancora dirci qualche cosa. Mi è stato chiesto di indagare $\mathrm{i}$ rapporti politici evincibili dallo sparuto scambio epistolare tra Gherardesca, moglie del conte Guido, signore di Battifolle, e Margherita di Brabante, ${ }^{2}$ moglie

1 Novati, L'epistola di Dante, pp. 508-509.

2 Pispisa, Margherita di Brabante; Falzone, Margherita di Brabante.

Federico Canaccini, Uninettuno

O Open Access. ( 2020 Federico Canaccini, published by De Gruyter. (@) BY This work is licensed under the Creative Commons Attribution 4.0 International License (CC BY 4.0). https://doi.org/10.1515/9783110590661-021 
dell'imperatore Enrico VII di Lussemburgo. Impresa non semplice per più di una ragione.

In primo luogo per l'esiguità del testo dei biglietti che la contessa scrive, per mano di Dante, all'imperatrice; esiguità nonché ripetitività, al punto che numerosi studiosi hanno ipotizzato che si trattasse di un'unica missiva, di volta in volta ritoccata. In secondo luogo per la difficoltà di valutare le relazioni politiche tra le parti in causa: il rapporto che lega l'imperatore alla intera famiglia Guidi, divisa al suo interno per rivalità intestine, per adesione più o meno antica all'idea di fedeltà all'Impero, ed infine - ed è il dato più importante - per la posizione più o meno condiscendente dei vari conti Guidi all'espansione della vicina Firenze. Da ultimo, l'impresa è complicata dal ruolo giocato dagli specifici attori dello scambio epistolare: la contessa e l'imperatrice (ma naturalmente anche i consorti, il conte Guido e Enrico VII). L’Alighieri, in quanto "mero estensore", non dovrebbe essere chiamato in causa per quanto attiene al contenuto delle epistole. Oppure, e sarà la parte conclusiva del mio intervento, Dante svolse un ruolo di diverso spessore che non quello di semplice scrivano di corte?

\section{Il trittico Battifolle}

A causa della loro brevità, le epistole VIII-X, attribuite all'Alighieri, hanno sollevato nel corso del tempo diverse questioni, compresa quella della paternità dantesca. A cavaliere del XIX e XX secolo, infatti, ci fu una sorta di "epidemico scetticismo" verso le opere attribuite al poeta fiorentino. Furono definite da autori quali tal Canonico Moreni «roba scritta in stile barbarico, con parole non rinvenibili neppure nel Ducange» opere quali il De vulgari eloquentia, la Questio e persino la Monarchia, definita da Prompt «this barbarous and abominable book». ${ }^{3}$ Novati criticò questo atteggiamento definendolo «incredulità aprioristica e scetticismo elevato a sistema». Tra le varie opere considerate spurie, le epistole erano particolarmente attaccabili e, all'interno del corpus epistolare, il "trittico Battifolle" ancor di più: esso infatti era stato scritto da Dante, ma a nome di qualcun altro. Nelle tre epistole non compare mai il nome dell'Alighieri, né vi è alcun indizio che ci lasci supporre che sia lui l'estensore di tali missive. Come indagare dunque? Se sulla base di questi argomenti molti studiosi avevano rifiutato l'idea che tali letterine fossero opera del fiorentino, si deve in parti-

3 Moore, The "Battifolle" Letters. 
colare a Scheffer-Boichorst $(1882)^{4}$ e Corrado Ricci (1891) la spinta a mantenerne la paternità dantesca. ${ }^{5}$

L'indizio principe per l'attribuzione a Dante ce lo fornisce la loro posizione all'interno del manoscritto Vaticano Palatino Latino 1729, l'unico che ce le ha preservate, giacché, come ha notato il Moore, ${ }^{6}$ esse non sono sistemate al principio o in chiusura della sezione epistolare, ma proprio al suo centro. L'ordine con cui il manoscritto ci presenta le nove epistole attribuite a Dante, a quanto pare scandito dall'importanza politica dei destinatari, è infatti il seguente: 1) Ep. VII; 2) Ep. VI; 3-4-5) Ep. VIII-IX-X (il “trittico Battifolle”); 6) Ep. II; 7) Ep. IV; 8) Ep. I; 9) Ep. V.

Particolare non trascurabile è la relativa prossimità con l'Alighieri del manufatto suddetto, giacché il manoscritto fu composto solo 70 anni dopo la morte del poeta. Al momento della realizzazione del codice, nel 1394, il copista non ebbe dubbi sulla paternità dantesca del “trittico Battifolle”. Doveva esserci perciò una tradizione precedente (a noi non pervenuta) che garantiva come dantesche le tre epistole. ${ }^{7}$ Benché le lettere non siano precedute da alcuna nota esplicativa o commento e non presentino dunque alcun nesso con l'Alighieri, la loro posizione e il fatto stesso che il copista non abbia avvertito la necessità di postillarle potrebbe già di per sé avvalorarne parzialmente l'autenticità. Di contro, si deve comunque sottolineare che nelle lettere non appare effettivamente alcun legame con la figura di Dante e, addirittura, la lettera X risulta firmata da terzi, ossia dalla contessa Gherardesca di Battifolle. ${ }^{8}$

Altri indizi della paternità dantesca sono alcuni riscontri di tipo formale $\mathrm{e}^{9}$ la datatio topica e cronologica del terzo biglietto, l'unico ad essere stato datato Poppi, 18 maggio 1311 («missum de castro Poppii XV Kalendas Iunias, faustissmi cursus Henrici Cesaris ad Ytaliam anno primo»); si tratta di una data vicina a quelle delle Ep. VI, inviata ai Fiorentini intrinseci il 31 marzo 1311, e della Ep. VII, indirizzata all'imperatore Enrico VII il 17 aprile 1311 da una località «sub fonte Sarni ${ }^{10}$ - forse dal castello di Porciano, ai piedi del Falterona, presso

4 Scheffer-Boichorst, Aus Dante's Verbannung.

5 Ricci, L'ultimo rifugio.

6 Moore, The "Battifolle" Letters.

7 Per quanto attiene alla questione del manoscritto e del suo autore, rinvio alle illuminanti relazioni di E. Romanini e A. Bartoli-Langeli contenute in questo medesimo volume.

8 Il nome della mittente è indicato nel manoscritto dalla sua iniziale (una G). Nell'edizione che fece il Giuliani, l'iniziale fu scambiata per una C. e l'identificazione della mittente cadde su Caterina, moglie di un altro conte Guido, inopinatamente anch'egli signore di Battifolle e coevo del suo omonimo.

9 Moore, Studies in Dante, vol. IV, pp. 256-75 e 287.

10 Con l'idronimo "Sarno" l'Alighieri identifica spesse volte nelle epistole il fiume Arno, pur conoscendo, come attesta il Boccaccio, il fiume campano, a lui noto almeno tramite Virgilio 
altri conti Guidi. ${ }^{11} \mathrm{Su}$ questi particolari topografici si tornerà nel prosieguo del discorso. $^{12}$

Novati, rimpiangendo la perdita di altri documenti epistolari danteschi ben più significativi di questi, ne parlò come di documenti «di sì tenue valore, che nulla o ben poco ci giova il possederle». ${ }^{13}$ Meno severo Mazzoni il quale, pur ammettendo che rispetto alle altre epistole queste certamente sono «meno solenni», le definì «assai ben architettate e clausolate», mentre Pastore Stocchi le ha riconosciute come dotate di «un'abile tessitura retorica» pur nella povertà contenutistica. $^{14}$

La brevità, la somiglianza tra i tre bigliettini e il fatto che la datazione sia presente nell'ultima delle tre epistole hanno indotto alcuni studiosi a ipotizzare che si tratti dello stesso testo, ${ }^{15}$ di cui sarebbero sopravvissute delle redazioni preparatorie (per lo più identificate nelle Ep. VIII e IX) e la redazione definitiva, che è anche l'unica a riportare la datazione $(E p . \mathrm{X}) .{ }^{16}$ Altri studiosi hanno invece ritenuto autentiche e autonome le tre epistole, discutendone fra l'altro anche l'ordine di invio e la relativa cronologia: ${ }^{17}$ Chiappelli propose un preciso ordine di stesura, assegnando ai primissimi del 1311 l'Ep. IX (forse addirittura anteriore all'Ep. VII), poi la X e infine l'VIII, caratterizzata, secondo lo studioso, da «una atmosfera più autunnale» di disincanto. ${ }^{18}$

\section{2 «Progenies maxima tuscanorum»}

Tra i vari nobili su cui Enrico VII pensava di fare affidamento non mancò la famiglia comitale guidinga, "progenies maxima tuscanorum», un tempo compattamente leale all'Impero ma, a seguito della divisione familiare avvenuta dopo Guido Guerra III, spezzata in vari rami tra loro in conflitto. ${ }^{19}$ Dal secondo

(Aen. VII, 738) e Lucano (Phars. II, 424). Sulla scelta di ribattezzare l'Arno col nome di Sarno cfr. Brugnoli, Sarno.

11 «Scriptum in Tuscia sub fonte Sarni XV Kalendas Maias, divi Henrici faustissimi cursus ad Ytaliam anno primo».

12 Imago et descriptio Tusciae. Colgo qui l'occasione per ringraziare l'amico e collega P. T. Stella per l'inaspettato ma provvido e graditissimo aiuto.

13 Novati, Le Epistole, p. 17.

14 Mazzoni, Le epistole di Dante, pp. 47-ss.

15 Padoan, La pubblicazione casentinese; Ep. (Pastore Stocchi), p. 68 e 74.

16 Rossetto, Per il testo critico, p. 105.

17 Mazzoni, Le epistole di Dante, pp. 47-ss.

18 Chiappelli, Osservazioni sulle tre epistole, pp. 558-ss.

19 La lunga storia di una stirpe comitale. 
matrimonio, contratto con Gualdrada di Bellincione Berti dei Ravignani, ${ }^{20}$ Guido ebbe infatti cinque figli maschi; secondo la tradizione longobarda, i quattro superstiti, al momento del raggiungimento della maggiore età, si spartirono il patrimonio paterno, dando vita a quattro rami identificabili dai nomi dei castelli in cui i vari conti presero dimora: da Guido VIII si ebbe il ramo di Battifolle (e di Bagno), da Marcovaldo quello di Dovadola, da Tegrimo il ramo di Modigliana (e Porciano), da Aghinolfo quello di Romena.

Nel corso del Duecento si assiste all'espansione del Comune di Firenze anche su territori guidinghi nei pressi dell'area urbana e, di converso, a un arretramento montano dei conti in Casentino: se Poggibonsi diventa fiorentina, i Guidi espandono i propri domini nella angusta valle casentinese, fondando in pieno XIII secolo addirittura nuovi fortilizi, come quello di Montemignaio, a difesa della alta valle del Solano-Scheggia. La frammentazione patrimoniale, le divisioni personali e politiche fra parenti, nonché il mutato quadro politico dopo il prevalere guelfo e fiorentino, avevano sostanzialmente cristallizzato la situazione geopolitica dei Guidi di fine Duecento. Per questo non ci saranno più nuove acquisizioni e i conti saranno via via estromessi da altre ed eterogenee forze, entrando in conflitto con i Camaldolesi, con il vescovo di Arezzo e il comune di Firenze, divenendo via via, "Signori delle montagne». ${ }^{21}$ Se il dominato si restringeva e si paralizzava sul Casentino, di converso si animarono le rivalità in seno alla famiglia.

Con la discesa di Federico II, agli inizi del Duecento, alcuni conti si mantennero su posizioni filoimperiali legandosi alla fazione ghibellina, mentre altri si dichiararono favorevoli al comune di Firenze e alla sua posizione guelfa, in chiave anti-imperiale. ${ }^{22}$ Sessanta anni dopo la morte dello Stupor Mundi, all'avvento di Enrico VII, nuove lotte fratricide scoppiarono all'interno di ciò che rimaneva della famiglia comitale. In primo luogo ci interessano i due fratelli, Guido Novello e Simone, signori di Poppi: costoro, dopo aver agito concordemente nelle fila ghibelline, una volta sconfitti dovettero scendere a patti con Firenze. Guido Novello rigettò gli accordi, mentre il fratello ne firmò altri separatamente il 28 agosto 1274. Da questa divisione si originarono anche i due rami di Bagno e di Battifolle, il primo facente capo al figlio di Guido Novello, Guglielmo Novello, e il secondo a Guido figlio di Simone, il marito della Gherardesca per conto della quale sono scritte le epistole.

Proviamo però adesso a distinguere i vari rami della famiglia dei Guidi, assegnando loro i rispettivi principali fortilizi casentinesi e "fotografando", per

$20 \mathrm{Su}$ questo personaggio cfr. Faini, Ruolo sociale, p. 9.

21 Wickham, The Mountains.

22 Canaccini, Ghibellini e Ghibellinismo. 
gli anni che interessano, una posizione o una simpatia politica che, talvolta, risulterà quanto mai "sfocata".

Con una buona approssimazione possiamo identificare il dominio del conte Guido di Battifolle, a questa altezza cronologica di fede fiorentina e filo-guelfa, con Poppi, Borgo alla Collina, Larniano e Fronzola, Quota, San Martino e Riosecco. Spostandoci verso l'Alto Casentino troviamo altri possessi del conte da Battifolle: il castello omonimo, oggi scomparso, e poi i dominî di Caiano e Montemignaio. Di posizione ghibellina invece suo cugino Guglielmo Novello, che dominava su Castel san Nicolò, Pagliericcio, Cetica, ma anche Farneta e Soci. Pratovecchio e Castel Castagnaio erano di proprietà del guelfo Guido Selvatico da Dovadola, mentre era di tradizione ghibellina il conte Guido Novello II, figlio di Federico di Guido Novello I, che dominava su Raggiolo, Ortignano, Garliano e San Piero. Di posizione incerta, perché di un guelfismo recente e traballante, i conti di Romena, quelli ricordati da Dante nel canto di Mastro Adamo, padroni anche di Lierna, Ragginopoli e Partina. Infine, ai piedi del Falterona, e quindi alle sorgenti dell'Arno, «sub fonte Sarni», incontriamo alcuni dei possessi casentinesi dei figli di Guido di Modigliana: Porciano in testa e poi Urbech, Papiano, Lonnano e il mercatale di Stia.

Il nobile e prolifico Guido di Modigliana e Porciano ebbe ben undici figli, di cui otto maschi. La posizione politica di questa famiglia appare chiaramente filo-ghibellina, ${ }^{23}$ anche se ben presto al suo interno sorgono delle screziature e persino delle ostilità. Alla discesa dei messi di Enrico VII, nel 1310, il conte Tegrimo di Guido di Modigliana li accolse a San Godenzo, assieme al fratello

23 Nel 1275 essi combattono assieme a Guido da Montefeltro contro i Guelfi bolognesi nella battaglia di Ponte San Procolo. Poco dopo, con il padre, accolsero il messo di Rodolfo d'Asburgo e nel 1277 sconfissero nuovamente i Guelfi bolognesi a Bagnacavallo. Tra i vari fratelli, Tegrimo sembra prediligere la residenza di Modigliana, di cui sembra il principale custode. Nel 1284 Tegrimo si trova in Valdambra (dove crea una potestas, definendo quell'area vicecomitatus) per ottemperare alla richiesta pontificia di allontanarsi per un periodo proprio da Modigliana. Dopo la battaglia di Campaldino, il conte Tegrimo sembra frequentare maggiormente invece la residenza di Porciano, dove erano rimasti i figli di un altro fratello (Corrado). Doveva risiedere con lui anche il fratello Tancredi, che viene condannato dal Comune di Firenze per avere, proprio in Porciano, depredato un mercante anconetano e deriso dei messi fiorentini. Dal 1299 è probabile che Tancredi resti a Porciano da solo, giacché Tegrimo viene nominato podestà di Faenza. Nel 1302 i capi dei Bianchi e dei Ghibellini tengono un convegno presso il castello dei Guidi di Modigliana a San Godenzo. Parteciparono a tale convegno Vieri dei Cerchi e altri due membri della sua famiglia, quattro Ubertini, Guglielmo dei Ricasoli, Andrea dei Gherardini, Bettino dei Pazzi, uno degli Scolari e quattro degli Uberti, con il nipote di Farinata, Lapo, in testa. Cfr. Pirillo, La signoria dei conti Guidi; Davidsohn, Storia di Firenze, vol. IV, pp. 319-320. 
Tancredi, ${ }^{24}$ colui che si mostrerà fedele sino alla fine all'imperatore di Lussemburgo. Sarà questo Tancredi, il signore di Porciano (e non Tegrimo), ad accompagnare il re a Roma per l'incoronazione, per poi affiancarlo nell'assedio di Firenze: nel 1313, perciò, Enrico concesse a lui i beni di Ildebrandino Guidi di Romena, da poco defunto, e altri beni che aveva espropriato a quei conti di Modigliana che non gli erano stati fedeli. ${ }^{25}$ La questione si sarebbe protratta nel tempo, e solo nel 1316 si tentò di addivenire ad una pace tra Tancredi e i figli del fu Tegrimo di Modigliana, Gualtieri, Fazio e Guido: in quella occasione le due parti schierarono i propri alleati, mostrandoci la portata e la qualità degli schieramenti. ${ }^{26}$ Tancredi poteva contare sull'appoggio dei conti Alberti di Mangona e sui fiorentini Cerchi e Tosinghi, mentre dal lato dei figli di Tegrimo erano Guido Novello di Federico, di Raggiolo, altri tra fratelli e figli di Tegrimo, due figli del conte Guglielmo Novello, il vescovo di Arezzo Guido Tarlati, e altri membri della aristocrazia aretina (Pietramala, Pazzi, Della Faggiola, Valenzano, Montedoglio), nonché Tommaso Donati, figlio di Corso.

Per comprendere le ragioni che collegavano un signore di salda tradizione ghibellina come Tancredi a fiorentini di solidi ascendenti guelfi come Cerchi e Tosinghi, concentriamoci su quest'ultima famiglia che consente di seguire più da vicino alcune dinamiche interfamiliari. Dopo la morte di Bindo Tosinghi a Campaldino (1289), suo figlio Baschiera, pur essendo di provata fede guelfa, fu escluso, assieme ad altri parenti, dagli uffici politici per opera dell'ambizioso e invidioso Rosso della Tosa (1309†) il quale, come scrive il Davidsohn «lo aveva sempre tenuto lontano da tutti gli uffici pubblici come un temibile rivale», al punto da indurlo ad allearsi coi Cerchi. Quando infatti Rosso, alla fine del Duecento, si schierò coi Donati, Baschiera prese per reazione posizioni cerchiesche. Da allora la famiglia dei Della Tosa fu divisa tra le due parti: nel maggio del 1300 Baschiera fu confinato a Sarzana insieme ad altri Bianchi - anche per volere di Dante, che allora era priore - ma poi, liberato da quella pena, l'11 novembre del 1300 si recò a Roma presso Bonifacio VIII per trattare la revoca dell'interdetto che gravava su Firenze. In quella medesima ambasciata vi era anche Betto de' Frescobaldi, già nemico di Giano della Bella, il quale, unico tra i membri del suo casato, parteggiava per i Cerchi dovendo loro una grande somma di denaro. L'anno seguente Baschiera Tosinghi rifiutò le offerte di pace

24 Il palazzo di San Godenzo era detto "Palazzo dello Specchio", forse per le finestre vetrate, assai rare per l'epoca. Nel 1306 tale residenza risulta ancora di Tancredi, come appare nella continuazione della Cronica Cantinelli; cfr. Davidsohn, Storia di Firenze, III, pp. 319-320.

25 Circa l'investimento politico ed economico dei conti Guidi rimasti di fede ghibellina alla venuta di Enrico VII si legga Nelli, Una corte feudale.

26 Notarile antecosimiano, 9497, c. 26r, 5 ottobre 1316. 
avanzate dai Neri e proseguì a combatterli sino alla morte, partecipando tra l'altro nel 1304 alla cosiddetta battaglia della Lastra. ${ }^{27} \mathrm{Al}$ momento della discesa di Enrico VII Baschiera non rimase in disparte: ${ }^{28}$ schierato davanti alle mura di Firenze nell'assedio portato dall'imperatore, figura quale “testis” all'emanazione delle condanne pronunciate dal tribunale imperiale. ${ }^{29}$

Il legame visibile nel 1316 tra Tancredi Guidi e i Tosinghi aveva quindi dietro di sé una certa storia. Sarà perciò da tenere bene a mente che, accanto a Tancredi, signore di Porciano in questi anni, figuravano membri dei Cerchi e molti di coloro che riponevano speranze in Enrico VII (Bianchi o Ghibellini che fossero) e che avevano già giocato le proprie carte, esponendosi alla fallimentare battaglia della Lastra e subendo il perpetuarsi dell'esilio, se non l'inasprimento della pena.

\section{3 «Plus guelphi quam guibelini, meo judicio»}

La discesa dell' «alto Arrigo» rinnovò ovunque antiche tensioni In Toscana, poiché Firenze sin dall'inizio si mostrò particolarmente fredda nei confronti dell'imperatore, l'impresa arrighiana mise in difficoltà comuni e nobili, che spesso si schierarono a seconda dei rapporti che avevano con questa città. ${ }^{30}$ In Italia si comprese che il nuovo sovrano, a differenza dei suoi predecessori, era intenzionato ad intervenire nelle faccende della penisola, e perciò Bianchi e Ghibellini inviarono degli ambasciatori tanto a lui quanto a papa Clemente V. Sappiamo che raggiunsero la Germania Guido di Filippo dell'Antella, legato alla compagnia degli Scali e socio di Giovanni de' Cerchi, e Ugolino da Vico, un ghibellino fiorentino che nel 1309 già si trovava a Spira per la Dieta imperiale.

Quando il 3 luglio 1310 si presentarono in Toscana gli ambasciatori del re, la risposta del Comune fiorentino fu più arrogante che evasiva. L'ambasciata imperiale era composta dal conte Luigi di Savoia, da Filippo vescovo di Eichstätt, da Gerardo, vescovo di Basilea, dal giurista Bassiano de' Gaschi e da Simone de' Reali. Essi protestarono per gli attacchi portati da Firenze contro la città di Arezzo e chiesero l'immediato ritiro delle truppe. Successivamente, quando

27 Viene condannato alla decapitazione il 5 aprile del 1302, come risulta dal Libro del Chiodo. 28 Figurerà anche per questo nella cosiddetta "Riforma di Baldo di Uguglione", la lista di circa 1500 personalità ghibelline o guelfe bianche escluse dal provvedimento di amnistia varato dal comune fiorentino (2 settembre 1311) per aver progettato o perpetrato atti di ostilità contro Firenze. Come è noto, anche l'Alighieri patisce lo stesso destino del Tosinghi.

29 Tarassi, Della Tosa, Baschiera.

30 Bowsky, Henry VII in Italy; Cognasso, Arrigo VII; Somaini, Henri VII. 
re Enrico poté valutare l'intero groviglio degli eventi, comprese che il primo segno della rivolta contro l'Impero era stato proprio l'atteggiamento di Firenze nei riguardi dei propri ambasciatori. ${ }^{31}$ Spostatisi nell'aretino, due degli ambasciatori ricevettero il giuramento di fedeltà dal conte Aghinolfo da Romena, già alleato se non addirittura capitano dei Guelfi bianchi. ${ }^{32}$

Il 30 settembre dello stesso anno, di contro, i fiorentini ricevettero re Roberto d'Angiò e "gli fu fatto grande onore, e armeggiata, e presenti grandi di moneta, e dimorò in Firenze infino a XXIIII dì d'ottobre per riconciliare i Guelfi insieme, ch'erano divisi per sette intra lloro, e per trattare al riparo dello 'mperadore». ${ }^{33}$ Solo nel novembre i Fiorentini preparano, peraltro senza inviarli mai, degli ambasciatori per Enrico. ${ }^{34} \mathrm{Si}$ danno invece da fare per erigere steccati e scavare fossi intorno alle mura: il 30 novembre «per tema della venuta dello 'mperadore sì ordinarono a chiudere la città di fossi (...) e poi, da la porta di San Gallo infino a quella del Prato d'Ognesanti, erano già fondate le mura, sì le feciono innalzare VIII braccia. E questo lavoro fu fatto sùbito e in poco tempo». ${ }^{35}$ L'atteggiamento anti-imperiale di Firenze, benché Enrico fosse stato coronato paradossalmente con egida papale, è quanto mai eloquente. A confermarlo vi fu, nel 1311, la stipulazione di una lega quinquennale anti-imperiale con Lucca, Siena, Bologna, Perugia e il re di Napoli. Infine, simbolo di un guelfismo intransigente e al limite dello scaramantico, fu la grande festa riservata il 13 aprile alle reliquie di san Barnaba, divenuto celeste protettore di Firenze e della Lega guelfa, dopo la vittoria di Campaldino: le reliquie «mandò da corte di papa il cardinale Pelagrù al Comune di Firenze, perché sapea che' Fiorentini l'aveano in grande devozione. E fune fatta in Firenze grande reverenza e solennità e furono riposte nell'altare di Santo Giovanni». ${ }^{36}$ Probabilmente non sarà stata una scelta casuale l'invio dei resti di quell'apostolo che aveva procurato la vittoria contro i Ghibellini proprio nel momento dell'arrivo di un nuovo imperatore. Nello stesso anno, il 2 settembre 1311, i guelfi «trassono di bando tutti i cittadini e contadini guelfi di che bando si fosse, pagando certa piccola gabella: feciono più ordini di leghe in città e in contado e coll'altre terre guelfe di Toscana». ${ }^{37}$ Come è noto, l’Alighieri, pur di famiglia e posizione guelfa, fu escluso con altri da tale amnistia.

31 Davidsohn, Storia di Firenze, III, pp. 524-527.

32 Acta Henrici VII Romanorum Imperatoris, I, n. 21 e Henrici VII Constitutiones, 4, 1, p. 307. $\mathrm{Su}$ Aghinolfo cfr. il contributo di M. Tavoni in questo stesso volume.

33 Villani, Nuova cronica, vol. II, p. 216.

34 Davidsohn, Storia di Firenze, III, p. 529.

35 Villani, Nuova cronica, vol. II, pp. 218-219.

36 Villani, Nuova cronica, vol. II, p. 222.

37 Villani, Nuova cronica, vol. II, p. 224. 
Ai fini di questo contributo e dell'ipotesi che sto tentando di proporre, una fonte risulta per noi particolarmente preziosa. Si tratta della Relatio de Heinrici VII imperatoris itinere italico composta dal vescovo di Butrinto, il domenicano Nicola de Ligny, che ci offre un quadro sull'atteggiamento politico di alcuni membri della famiglia Guidi, e in particolare del signore da Battifolle, all'altezza della discesa dell'imperatore, ed in particolare nell'ottobre del 1311. ${ }^{38}$

Questo nuovo vicario, assieme al notaro papale Pandolfo Savelli, dopo aver attraversato gli ostili territori di Bologna e Firenze e aver rischiato più volte la vita, fu scortato dal conte Tegrimo di Modigliana: la notte tra il 27 e il 28 ottobre li condusse a San Godenzo e poi fino in Casentino, dove il prelato incontrò due fratelli di Tegrimo, Tancredi - che evidentemente in questo periodo vive in Casentino - e Bandino. ${ }^{39}$ Successivamente incontrarono anche il conte Ruggero figlio di Guido Salvatico di Dovadola: ${ }^{40}$ «isti omnes dicuntur de comitibus Guidonibus et sunt comites palatini; et eiusdem domus sunt comes de Batefole et comes Salvaticus [cioè il conte di Dovadola]. Item comites de Romena omnes vocant se comites palatinos et magnos redditus habent in Romandiola». In realtà questi Guidi in comune avevano oramai poco più che il nome e qualche porzione di castello, e se ne era avveduto anche il vescovo di Butrinto, giacché segnalò che

inter istos quidam sunt guelphi et illi sunt ditiores, videlicet comes Salvaticus et comes de Batifole et comes Bandinus [il vescovo di Arezzo]. (...) Omnes juraverunt nobis fidelitatem et venire ad dominum suum personaliter, vel mittere, si impediti essent, statim quod esset in Tuscia, et ipsum associare ad coronam in illa decentia in qua possent secundum suam facultatem. Isti omnes nos multum honoraverunt et gaudenter receperunt, et plus guelphi quam guebelini, meo iudicio.

Quindi, se identità politica aveva, il conte di Battifolle l'aveva guelfa, almeno a detta del messo imperiale.

38 Nikolaus von Butrinto, Relatio de itinere Italico.

39 Nikolaus von Butrinto, Relatio de itinere Italico: «altera die, que fuit sanctorum Symonis et Jude vigilia in tertiis, predicti tres dimiserunt nos in quodam oppido in quo Florentini et comes Tegrinus habebant dominium. Et in nocte dictus comes Tigrinus duxit nos inter Alpes ad unum burgum suum qui vocatur burgus Sancti Gaudentii et mutuavit nobis equos quot potuit pro familia, que erat pedes. Postea ivimus cum eo ad alium locum; ubi ad nos venerunt duo fratres dicti comitis Tegrini, videlicet comes Tancredus et comes Bandinus. Postea invenimus quartum fratrem comitem Rogerium» (p. 525).

40 Ruggero, ulteriore fratello di Tegrimo e Tancredi, non avendo mantenuto gli aiuti promessi agli ambasciatori di Enrico VII in San Godenzo, si vedrà confiscati tutti i suoi beni, che saranno assegnati proprio a Tancredi, col quale entrerà in guerra sino alla sua morte (1318). 
Nella primavera del 1312 a Pisa converge il fior fiore del ghibellinismo toscano: ${ }^{41}$ vi sono anche numerosi membri dei Guidi di Modigliana, tra cui certamente Tancredi, c'è il conte Aghinolfo da Romena e suo fratello, il vescovo Ildebrandino, che proprio a Pisa morirà in quei giorni. ${ }^{42}$ A Pisa vi sono forse anche Dante e un giovanissimo Petrarca di appena otto anni, se è vera l'informazione che lo stesso poeta scrisse anni dopo a Giovanni Boccaccio. ${ }^{43}$ Ma non vi sono i conti Guidi dei rami di Battifolle e di Dovadola che hanno già ripiegato su Firenze:

non obstantibus fidelitatibus, nullus de istis Guelphis venit ad eum in Tusciam vel misit ad renovandum juramentum, sed dilationem petiverunt et habuerunt usque ad terminum petitum, et in termino petito, iam imperatore existente ante Florentiam, confederati sunt publice cum Florentinis, cum ipsis in Florentia existentes. ${ }^{44}$

Dalle parole del messo domenicano risulta perciò chiaramente la differenza di schieramento politico dei vari membri della famiglia guidinga: per quanto essi avessero accolto, promesso e giurato fedeltà a Enrico, in molti gli risultarono «plus guelphi quam guibelini» e, al momento di esporsi, furono ben pochi quelli che confermarono la loro sincera devozione per l'imperatore. Tra questi, concentrando il nostro interesse sui pochi nobili che attirano la nostra attenzione, non certo il conte Guido di Battifolle, bensì il conte Tancredi di Porciano.

\section{4 «Sub fonte Sarni»}

Il 31 del mese di marzo del 1311 Dante indirizzava, da un luogo imprecisato probabilmente del Casentino giacché «sub fonte Sarni», la celebre epistola VI agli "sceleratissimi fiorentini”, in cui li accusava di cieca stoltezza nell'opporsi alla volontà divina di un nuovo pacificatore. Sull'identificazione del luogo da cui sono state inviate le epistole VI e VII si è molto discusso. Sulla base di ciò

41 Nikolaus von Butrinto, Relatio de itinere Italico: «qui erant Guebelini inter istos vel venerunt qui potuerunt vel miserunt et cu meo fuerunt usque ad mortem suam» (p. 526).

42 Il vescovo Ildebrandino Guidi dovette essere il principale promotore della calorosa accoglienza che la città di Arezzo riservò agli ambasciatori imperiali nel 1311. Successivamente li accolse nel castello di Civitella, prestando giuramento di fedeltà. In cambio, Enrico fece nominare Ildebrandino suo vicario in Arezzo. Nella primavera del 1312, quando Enrico giunse in Toscana, il presule si recò a Pisa per presentare omaggio e avere la conferma del titolo vicariale. Cfr. Bicchierai, Ildebrandino Guidi.

43 Ceccarelli Lemut, Ghibellini e guelfi bianchi, nota 85.

44 Nikolaus von Butrinto, Relatio de itinere Italico, pp. 525-526. 
che si è visto finora, credo che si tratti del castello di Porciano dove, come ho ipotizzato poco sopra, doveva risiedere in questi anni Tancredi, colui che, tra i conti di Modigliana, si rivelerà il più fedele seguace di Enrico VII, e con cui Dante ha forse già condiviso più di un sogno e di una delusione.

A ciò si aggiunga la clausola «sub fonte» che va intesa, secondo me, come 'ai piedi della sorgente' e non come un generico riferimento alla vasta valle del Casentino, dove scorre il primo tratto dell'Arno. Il maniero di Porciano è il primo castello, dei tanti fortilizi guidinghi, che il fiume incontra lungo il proprio corso: esso dista appena 7 kilometri, in linea d'aria, da Capo d'Arno, e si erge proprio ai piedi del monte Falterona. Si tenga a mente poi che l'epistola VI riporta l'ulteriore specificazione di «in finibus Tuscie», e infatti Porciano si trova all'estremo confine della valle casentinese: oltre il crinale vi è la brulla Romagna. ${ }^{45}$ Se Dante avesse voluto far riferimento a Poppi, che dista circa altri 10 kilometri da Porciano, non avrebbe, a mio avviso, adottato una locuzione come «sub fonte». Inoltre, nel noto canto XIV del Purgatorio, il poeta sa bene che «per mezza Toscana si spazia / un fiumicel che nasce in Falterona» (Purg. IV, 16-17). E, se si accetta l'ipotesi di Casini e Barbi, il "porci” che compare pochi versi dopo («tra brutti porci, più degni di galle / che d'altro cibo fatto in uman uso, / dirizza prima il suo povero calle», vv. 43-45) sarebbe stato apposto come riferimento proprio al castello di Porciano. ${ }^{46}$ Quando invece si trova presso Gherardesca, l'Alighieri scrive esplicitamente che la lettera è stata inviata il 18 maggio «de Castro Poppii». ${ }^{47}$

Si tratta ora di concentrarci finalmente sul "trittico Battifolle" per provare a capire perché Dante scrivesse per conto di Gherardesca alla moglie dell'imperatore, per giunta da Poppi.

45 «Scripsit pridie Kalendas Apriles in finibus Tuscie sub fonte Sarni, faustissimi cursus Henrici Cesaris ad Ytaliam anno primo» (Ep. VI, 27).

46 Cfr. Lanci, Porco: «netto il valore figurato in Purg. XIV, 43, dove il termine indica gli abitanti del Casentino, che l'Arno incontra dapprima nel suo corso. Secondo le varie ipotesi dei commentatori, essi sono così chiamati o perché di costumi rozzi e primitivi o in riferimento al castello di Porciano, possesso dei conti di Modigliana, ramo ghibellino della casata dei conti Guidi, "che erano nei documenti fiorentini solitamente indicati come conti di Porciano" (CasiniBarbi). Pietro però, seguito, sostanzialmente, da Benvenuto, Landino, e da molti altri, scorgeva in brutti porci un'allusione alla lussuria dei "comites Guidos, adeo in opera venerea luxuriosa implicitos, quod porcis quodammodo adaptantur”. Quest'ultima motivazione, riproposta, sia pure in via ipotetica, anche da commentatori moderni (Scartazzini-Vandelli, Sapegno), è giudicata "piuttosto fantastica" dal Mattalia. È da ricordare, infine, che si è anche pensato da alcuni all'inimicizia dei conti di Modigliana nei confronti di Firenze e al fatto che essi non aiutarono sufficientemente i Bianchi fiorentini nei loro tentativi di rientrare in patria».

47 «Missum de Castro Poppii XV Kalendas Iunias, faustissimi cursus Henrici Cesaris ad Ytaliam anno primo» (Ep. X, 6). 
In primo luogo bisogna considerare che non doveva essere pratica inusuale che le consorti di sovrani, conti o alti dignitari, intrattenessero scambi epistolari col fine di cattivarsi simpatie o alleanze politiche, e avessero anche un qualche ruolo nelle missioni del marito. La contessa si fa promotrice della riverenza che il suo sposo dovrebbe avere nei confronti dell'imperatore, rivolgendo auguri all'imperatrice. La regina, del resto, appare presente e attiva nel corso della spedizione: sempre Nicola de Ligny vescovo di Butrinto ci informa, ad esempio, che «regina, piissima domina, (...) claves civitatis (si tratta di Cremona) receperat, et omnes de suo consilio dabant spem quam quod laborarent secundum suum posse, sic quod non debebant desperare». Oppure che, quando fu intercettato un messo che portava lettere da Brescia a Firenze inviate dal podestà di Brescia, «Pynus nomine», le lettere furono lette non solo dal re, ma anche dalla regina, cosi anche come dal vescovo «Treverensis, dominus Sabaudie, unus clericus qui vocatur magister Henricus de Jodonia, juris professor» e dallo stesso estensore della Cronaca, il vescovo di Butrinto. ${ }^{48}$

Si tratta tuttavia di provare a capire perché il poeta si trovasse proprio a Poppi: una domanda a cui non è semplice dare una risposta univoca e definitiva. Una possibilità è che egli fosse lì in qualità di messo imperiale. Nella $E p$. VII Dante dichiara di aver toccato e baciato i piedi del sovrano, forse a Milano, forse in un'altra città del Nord Italia: «io ti vidi benignissimo, come alla maestà imperiale si conviene, quando le mie mani strinsero i tuoi piedi, e le mie labbra pagarono il loro debito. Allora esultò lo spirito mio in me e dissi tacitamente: ecco l'Agnello di Dio, ecco colui che toglie i peccati del mondo» (Ep. VII, 9-10). Possiamo quindi ipotizzare, senza tuttavia alcuna certezza, che nel corso di questo incontro la regina abbia affidato al poeta un biglietto indirizzato a Gherardesca, e che questo biglietto abbia poi innescato lo scambio epistolare.

Il Petrocchi scartava l'ipotesi che l'Alighieri potesse essere stato inviato in Casentino dai sovrani sulla base del fatto che nell'epistola VII (la cui datazione è 17 aprile 1311 e che solo da Chiappelli è posta cronologicamente dopo la epistola IX) egli avesse affermato di scrivere «tam pro me quam pro aliis»: «non può essersi recato come messo di Enrico, ché anzi scrivendo "tam pro me quam pro aliis" mostra di esser portavoce di tutta la comunità degli esuli fiorentini» ${ }^{49}$. Da che cosa si evincerebbe che Dante rappresentasse tutta questa comunità di esuli se vi è un «pro aliis»? Inoltre, ed è l'argomento che qui interessa, l'epistola VII è di un certo tipo, il "trittico Battifolle riguarda eventualmente un altro tipo di missione. Infatti un conto è scrivere a proprio nome (e, supponiamo, a nome dei Bianchi e dei Ghibellini) all'imperatore; un conto è - ed è invece il

48 Nikolaus von Butrinto, Relatio de itinere Italico, pp. 509-511.

49 Petrocchi, Vita di Dante, p. 149. 
caso delle epistole VIII-X - recare eventualmente da parte dell'imperatrice una missiva alla Gherardesca e poi assumere il ruolo di suo segretario, a un mese di distanza dall'epistola ad Arrigo. Perciò non ravviserei in quel passo dell'epistola VII l'impossibilità che Dante sia stato inviato, limitatamente allo scambio del "trittico Battifolle", quale messo imperiale in Casentino al fine di prendere contatti con la sola contessa.

Altra ipotesi è che egli fosse lì come inviato degli esuli fiorentini. I Bianchi e i Ghibellini - forse gli stessi con cui condivide le intenzioni espresse nell'epistola VII, certamente gli stessi con cui condivideva l'esilio - all'arrivo di Enrico VII potrebbero aver chiesto al poeta di catechizzare quanti tra i Guidi erano ancora più titubanti nell'abbracciare la causa imperiale, abbandonando il guelfismo, Firenze e la sua cecità. Per questo non credo sia assurdo ipotizzare non tanto che Dante fosse ospite del conte Guido da Battifolle (guelfo nero e antiimperiale, legato a Bonifacio e a Corso Donati), quanto piuttosto che avesse trovato riparo a Porciano presso il conte Tancredi, di simpatie ghibelline e cerchiesche, come abbiamo visto. Ce lo confermerebbero le due epistole succitate, che furono date «sub fonte Sarni», con il loro contenuto chiaramente anti-fiorentino e filo-imperiale, e anche la posizione ghibellina e filo-bianca del conte.

Perché dunque trovare asilo presso il Guido di Battifolle in quei mesi? Certo è che l'epistola X sia stata inviata proprio dal castello di Poppi. Si può immaginare una missione di Dante finalizzata a convertire gli "incerti”, come i Guidi di Romena - che comunque avevano rafforzato i legami con Arezzo, appoggiando i Bianchi contro Firenze - o, come il signore di Poppi, più schiettamente anti-imperiali?

Guido Guidi era senza dubbio uno di questi incerti. ${ }^{50}$ La sua carriera ce lo mostra anzi come vero guelfo, anti-ghibellino e anti-imperiale, a dispetto di quanto dice la moglie nell'epistola $\mathrm{X}$, dove chiede che i sovrani si degnino «di guardare con gli occhi della mente la purezza di una fedeltà altre volte già delibata» $(E p . \mathrm{X}, 3)$. Quali siano questi segni di fedeltà resta assai poco chiaro: forse l'antico titolo di comes palatinus? Oppure la contessa, parlando in prima persona, si riferisce ai segni della propria famiglia, i della Gherardesca?

Questo Guido era figlio del conte Simone il quale, assieme a Guido Novello, guidò la famiglia comitale in senso ghibellino negli anni attorno a Montaperti. Dopo i disastri di Benevento e Tagliacozzo, però, i due fratelli imboccarono strade opposte: nel 1273, su iniziativa papale, entrambi furono costretti a stipulare una pace con Firenze che prevedeva la consegna in ostaggio dei figli di Guido Novello e di sedici abitanti di Poppi, nonché un giuramento da prestare agli

50 Bicchierai, Guidi, Guido. 
emissari di Carlo d’Angiò. Mentre tuttavia Guido Novello si ribellò alla pace, tradita dall'Angioino e dai Guelfi, il conte Simone e suo figlio rimasero fedeli a questa scelta e nel 1281, morto il padre almeno da un anno, Guido giurò la Pace del cardinal Latino. Podestà di Città di Castello, si distinse giovanissimo nell'assedio di Messina, in appoggio a Carlo d'Angiò, contro gli Aragonesi che combattevano "pro exaltacione predecessorum nostrorum»: ${ }^{51}$ gli Svevi. Qui si rivela, oltre all'anti-ghibellinismo, la sua fedeltà agli Angioini, e quindi la sua conseguente ostilità agli Svevi. Proseguì la collaborazione con Firenze, assediando Poggio Santa Cecilia, ricettacolo di Ghibellini sbanditi, fino alla capitolazione. Nel 1288 partecipò probabilmente alla guerra contro Arezzo (all'epoca retta da un governo ghibellino, e dove era presente il vicario imperiale Percivalle Fieschi). Non sappiamo se prese parte allo scontro di Campaldino (11 giugno 1289), dopo il quale fu indennizzato con 3000 fiorini d'oro per i danni subiti al castello di Poppi - di cui prese pieno controllo successivamente. Nei primi del Trecento lo vediamo addirittura appoggiare Corso Donati. Più di un fattore conduceva questo Guidi ad abbracciare la fazione nera: papa Bonifacio VIII lo aveva allettato offrendo un canonicato a un suo figlio, una sua figlia aveva sposato Musciatto dei Franzesi, consigliere di Carlo di Valois; inoltre uno dei suoi maggiori creditori era Manetto Scali, che si era schierato con i Bianchi. Com'è noto, il tentativo di Corso Donati di prendere il potere fallì temporaneamente e il conte fu condannato al bando; ma nel novembre del 1301 i Neri presero comunque il potere.

Se si accetta l'ipotesi che l'Alighieri fosse in "missione" in Casentino per conto di Enrico VII o dei Bianchi per far proseliti per la causa arrighiana, resta tuttavia incerto quanto Dante abbia potuto influenzare con la propria presenza i membri più incerti dei Guidi. ${ }^{52}$ Nelle righe delle epistole VIII-X, secondo Moore, sarebbe errato cercare tracce del pensiero dantesco, poiché Dante compose tali testi in qualità di segretario, e il dictator è chiamato naturalmente a rispettare volontà e contenuti del mittente. Però, specie nella chiusa della VIII e della X, non si può non notare qualcosa di squisitamente dantesco: la devozione per l'Impero e la divina missione di Enrico. Come conciliare allora il ruolo di secretarius con un contenuto filo-imperiale che appartiene più all'estensore che, come si è visto, certamente non al mittente? Come generica captatio benevolentiae? Forse c'è un'altra possibilità.

51 Canaccini, Ghibellini e Ghibellinismo.

52 La letteratura sul pensiero politico dell'Alighieri è naturalmente molto ampia. Si fa riferimento almeno a Solmi, Il pensiero politico; Ercole, Il pensiero politico; Passerin d'Entrèves, Dante politico; De Angelis, Il concetto; Lumia, Aspetti del pensiero politico; Mancusi-Ungaro, Dante and the Empire, 1987; Woodhouse, Dante and Governance; Carletti, Impero, stati particolari; Carletti, Dante politico. 
Si deve supporre infatti che Dante abbia rispettato le volontà della contessa (e forse del conte), che quelle siano perciò non le parole di Dante ma quelle della Gherardesca (e del suo consorte). ${ }^{53}$ Ma non credo sia un azzardo supporre che nei mesi trascorsi in Casentino le idee di un intellettuale del calibro dell'Alighieri fossero state fatte proprie da alcuni nobili casentinesi: sono i mesi in cui il poeta è mosso dalla ferma convinzione del trionfo imminente di Arrigo VII.

La parabola di Enrico VII si consumò tuttavia rapidamente e tragicamente. Nell'ottobre del 1311 i sovrani raggiunsero Genova. Tra il 13 e il 14 dicembre, «in palatio eredum Benedicti Zachariae», ${ }^{54}$ l'imperatrice Margherita morì a 34 anni, colpita dalla peste. In primavera Enrico arrivava a Pisa. Con ogni probabilità il conte Guido aveva a quel punto già deciso di tornare (se mai se ne era allontanato) alla sua tradizionale alleanza con Firenze, e così avevano fatto anche Guido Salvatico e suo figlio Ruggero di Dovadola. Sei mesi più tardi Enrico veniva incoronato in Laterano, e nel settembre del 1312 iniziava il lungo e vano assedio di Firenze. Il Bruni ci informa di un'altra lettera da cui traspariva la devozione di Dante per l'Impero, ma anche il rispetto per la propria città: «pure il tenne tanto la reverenza della patria che, venendo lo imperadore contro a Firenze e ponendosi a campo presso alla porta, non vi volle essere, secondo lui scrive, con tutto che confortatore fusse stato di sua venuta». ${ }^{55}$ Se con l'imperatore si schierò il conte Aghinolfo, Guido da Battifolle (assieme a Guido Salvatico e Ruggero di Dovadola) era a quel punto dalla parte dei fiorentini, a minacciare alle spalle, da Ganghereto, le forze imperiali. L'imperatore li condannò vanamente per fellonia. ${ }^{56}$ Un anno dopo, il 24 agosto 1313, anche l'imperatore moriva, colpito dalla malaria durante l'assedio di Siena. Enrico non aveva nemmeno 40 anni e le speranze per un effettivo potere imperiale, in Italia, morirono con lui. A scortarne la salma vi erano alcuni Guidi, tra cui il futuro leader dello schieramento ghibellino, tal Guido Novello il giovane, conte di Raggiolo, e Tancredi, signore di Porciano.

53 Un'ulteriore riflessione deve essere fatta circa la eventualità che il conte, nei giorni di permanenza in Poppi dell'Alighieri, non fosse neppure presente in Casentino. Ulteriori indagini sul Diplomatico di Firenze o sulle Provvisioni, potrebbero magari portare qualche informazione su una sua permanenza magari in città, in questi mesi. Che a scrivere sia la consorte e non il marito non è certo una prova a sostegno di questa ipotesi, ma faciliterebbe la presenza dell'Alighieri presso il maniero poppese. Sia chiaro che non sto proponendo un sotterfugio della contessa alle spalle del marito, che viene nominato esplicitamente; ma si deve riflettere almeno sul fatto che la comunicazione intercorra esclusivamente tra le due donne.

54 Mussato, De gestis Heinrici VII, col. 404.

55 Bruni, Vita di Dante, p. 237.

56 I conti Salvatico di Dovadola e Guido di Battifolle furono privati di tutti i feudi imperiali, restituiti ai proprietari solo il 4 gennaio del 1330 per volere di papa Giovanni XXII. Cfr. Davidsohn, Storia di Firenze, III, p. 713. 
Per Dante terminava la speranza di restaurazione dell'«alto imperio», di porre fine al suo esilio e tornare a Firenze. Il voltafaccia (qualora ve ne sia stato uno) del conte di Battifolle dovette frustrare ulteriormente i sogni di giustizia di Dante. Non sappiamo se il Guidi prese parte alla guerra contro i Bianchi e contro Dante: sappiamo però che, dopo la morte di Enrico VII, sarà proprio questo conte Guido a rivestire il ruolo di vicario di Roberto d'Angiò in Firenze per un anno, ottenendone il plauso, dopo la mala condotta del suo predecessore, nientemeno che del cognato del re, Bertrando del Baux. Di certo il poeta non era nelle grazie di re Roberto a causa delle evidenti posizioni filoimperiali che aveva preso con le lettere - e forse anche con la Monarchia che sarebbe stata invisa all'Angioino e da lui fatta più volte confutare. ${ }^{57}$

La delusione, per l'Alighieri, doveva essere completa.

57 Padoan, Tra Dante e Mussato, pp. 16-17. 
